\title{
Germ cell sex prior to meiosis in the rainbow trout
}

\author{
Mingyou Li ${ }^{1 *}$, Qian Shen ${ }^{1 *}$, Foong Mei Wong ${ }^{1}$, Hongyan $X^{1}{ }^{1}$, Ni Hong ${ }^{1}$, Lingbing Zeng ${ }^{2}$, Lin Liu ${ }^{2}$, Qiwei Wei ${ }^{2}$, \\ Yunhan Hong ${ }^{1,2 \bowtie}$
}

${ }^{1}$ Department of Biological Sciences, National University of Singapore, 10 Kent Ridge Crescent, Singapore 119260, Singapore

2 Yangtze River Fisheries Research Institute, Chinese Academy of Fisheries Sciences, 41 Jianghan Road, Jingzhou 434000, Hubei, China

$凶$ Correspondence: dbshyh@nus.edu.sg

Received December 8, 2010 Accepted December 27, 2010

\begin{abstract}
Germ cells make two major decisions when they move from an indeterminate state to their final stage of gamete production. One decision is sexual commitment for sperm or egg production, and the other is to maintain mitotic division or entry into meiosis. It is unclear whether the two decisions are made as a single event or separate events, because there has been no evidence for the presence of germ cell sex prior to meiosis. Here we report direct evidence in the fish rainbow trout that gonia have distinct sexuality. We show that dazl expression occurs in both male and female gonia but exhibits differential intracellular distribution. More strikingly, we show that boule is highly expressed in male gonia but absent in female gonia. Therefore, mitotic gonia possess sex, sperm/egg decision and mitosis/meiosis decision are two independent events, and sperm/egg decision precedes mitosis/meiosis decision in rainbow trout, making this organism a unique vertebrate model for mechanistic understanding of germ cell sex differentiation and relationship between the two decisions.
\end{abstract}

KEYWORDS boule, dazl, germ cell sex, gonia, meiosis, trout

\section{INTRODUCTION}

A multicellular organism comprises two major cell lineages, the soma and germline. The germline founder in diverse animals is specified during early embryogenesis as primordial germ cells (PGCs), which migrate into the developing gonad for the production of gametes (Wylie, 1999). Once in the gonad, germ cells make a first major decision for sexual commitment for sperm or egg production. At the stage of gamete production, germ cells make a second decision, namely the maintenance of mitotic division or entry into meiosis (Kimble and Page, 2007). Meiosis is a specialized cell division that occurs exclusively in germ cells. During embryonic development, germ cells increase their number by using the standard mitotic division that occurs also in somatic cells. They undergo mitosis also in adults for stem cell maintenance. Upon maturation, germ cells commence meiosis, which reduces the chromosome number from the typical two sets (diploidy) to a single set (haploidy) in sperm and eggs. A diploid state is restored at fertilization when a sperm and an egg are fused to form the zygote, the start point of a new life of the next generation. How germ cells make the two decisions have remained longstanding questions. It is even unclear whether the two decisions are made as a single event or separate events (Kimble and Page, 2007). There are two models. In one model, sperm/egg and mitosis/meiosis decisions are one and the same event, and a sex-specific regulator induces sex-specific entry into meiosis and commits the germ cell to differentiation as sperm or egg. In the other model, the two decisions are controlled by distinct regulators, and a sex-neutral mechanism induces meiotic entry but sexspecific regulators operate in parallel to generate sex-specific aspects of meiosis and differentiation as sperm or egg (Kimble and Page, 2007). The identification of a molecular sex marker for premeiotic germ cells will be critical to distinguish between the two models.

Sex is a mystery since long, because it is the basis of sexual reproduction in multicellular organisms including

"These authors contributed equally to this work. 
human. Although germ cells are the final decision maker and ultimate player to produce the male gametes sperm or the female gametes eggs, most studies on sex differentiation have so far focused on the sex of gonadal somatic cells (Kocer et al., 2009). Formation of testis-triggering Sertoli cells or ovary-triggering granulose cells determines the gonadal sex, which in turn determines the organismal sex and germ cell sexuality (Kocer et al., 2009; Uhlenhaut et al., 2009). In the fish medaka, the gonadal male sex is determined by dmy/dmrt1y (Matsuda et al., 2002; Volff et al., 2007). Paradoxically, with germ cells, animals usually develop into two opposite sexes, male and female; without germ cells, they produce only one sex, male, pointing to a conserved interplay between germ cells and gonadal sex (Kurokawa et al., 2007). Gonia within a testis (spermatogonia) or an ovary (oogonia) contain germ stem cells capable of self-renewal and differentiation even in culture (Hong et al., 2004). Transplantation data in fish support that gonia are sexually indeterminate or plastic (Okutsu et al., 2006; Okutsu et al., 2007; Yoshizaki et al., 2010). However, there is no evidence for the absence or presence of gonial sex (Kocer et al., 2009). Here we show in the fish trout that male gonia are daz $^{+} /$boule ${ }^{+}$, whereas female gonia are $d a z \Gamma^{+} /$ boule $^{-}$, providing direct evidence for germ cell sexuality prior to meiosis.

\section{RESULTS AND DISCUSSION}

We chose trout as a test-organism to study the sexuality of germ cells prior to meiosis, because in this organism, spermatogonia and oogonia have been demonstrated to take the male or female pathway according to the sex of a host gonad but irrespective of the sex of their origin (Okutsu et al., 2006; Yoshizaki et al., 2010). Recently, we have established that boule and dazl encoding RNA binding proteins of the DAZ family are germ cell markers in rainbow trout (Li et al., 2011). By using them and vasa as highly conserved germ cell marker also in rainbow trout (Xu et al., 2010), we performed in situ hybridization (ISH) to examine differential expression between male and female germ cells before and during meiosis.

In the adult testis, chromogenic ISH revealed that both boule and dazl were highly expressed in mitotic spermatogonia but undetectable in surrounding somatic cells (Fig. 1A and $\mathrm{B}$ ), conforming to our previous observation and demonstrating the specificity of the ISH procedure. We then performed fluorescence ISH and examined RNA expression patterns before and during meiosis. Meiosis represents the earliest stage so far in mammals that shows germ cell sexuality at the cellular level (Kocer et al., 2009). In the adult testis, male germ cells at premeiotic, meiotic and post-meiotic stages of development can be clearly seen on the basis of relative locations and morphology (Fig. 1C). The boule signal was intense in gonia (mitosis), spermatocytes (meiosis) and barely detectable in spermatids (post-meiosis), and distributed evenly in the cytoplasm of these cells (Fig. 1D). The expression of dazl was similar to boule in terms of developmental stages. However, we observed two salient features (Fig. 1E). One is the concentration of the dazl signal in several speckles in contrast to uniform boule distribution. The other is a relatively high level of expression in spermatids, in which dazl was found in the so-called chromatoid body (CB). Differences in stages and intracellular distribution became more evident upon merge (Fig. 1F). Thus, boule and dazl exhibit mitotic and meiotic germ cells expression, but possess different intracellular distribution in male.

In the young ovary, boule expression was absent in oogonia and became detectable in stage-I early oocytes, whereas dazl was easily detectable in both oogonia and oocytes and exhibited uniform cytoplasmic distribution (Fig. 2A), indicating the absence of boule expression in oogonia of immature female. In the adult ovary, absence of boule expression was also observed in oogonia, which displayed strong dazl expression (Fig. 2C-D). Interestingly, the boule signal was more abundant in the perinuclear region, whereas dazl distributed widely in the cytoplasm including the peripheral cytoplasm (Fig. 2D). These data suggest that boule expression is absent in oogonia of immature and mature females.

In rainbow trout, efforts have made toward the identification of several sex markers such as $d m r t 1$ and fox/2, which were found to show dimorphic expression at critical stages of gonadal development and sex differentiation (Baron et al., 2005; Vizziano et al., 2007). In these studies, sex-dimorphic expression of these genes was observed in the gonadal somatic cells. In the current study, we have revealed the differential expression of boule in male gonia and differential intracellular distribution of dazl transcript between male and female gonia in rainbow trout. Briefly, in rainbow trout, male gonia are $\mathrm{daz}^{+} / \mathrm{boule}^{+}$, whereas female gonia are $\mathrm{daz}^{+} /$ boule ${ }^{-}$instead. Furthermore, male gonia are daz ${ }^{\beta}$ (speckle $d a z l)$, whereas female gonia are $d a z l^{d}$ (disperse dazl). In conclusion, rainbow trout male gonia are $d a z l^{+\mathrm{s}} / \mathrm{boule}^{+}$, whereas female gonia are daz/ $/^{+d} /$ boule ${ }^{-}$(Fig. 3). In fish, the expression of boule, dazl and vasa has also been examined in medaka (Xu et al., 2009), a laboratory fish model for germ cell biology (Herpin et al., 2007; Li et al., 2009) and stem cell biology (Hong et al., 1996, 1998; Yi et al., 2009). Notably, the medaka dazl expression also exhibits differential intracellular distribution between male and female gonia (Fig. 3), indicating that gonial sexuality may not be limited to rainbow trout. Our data provide direct evidence in a fish that germ cells prior to meiosis possess an established sexuality as male or female gonia, instead of being sexually indeterminate. Our data also raise an interesting question as to whether boule and dazl are merely cellular markers or even important regulators of gonial sexuality in rainbow trout. 

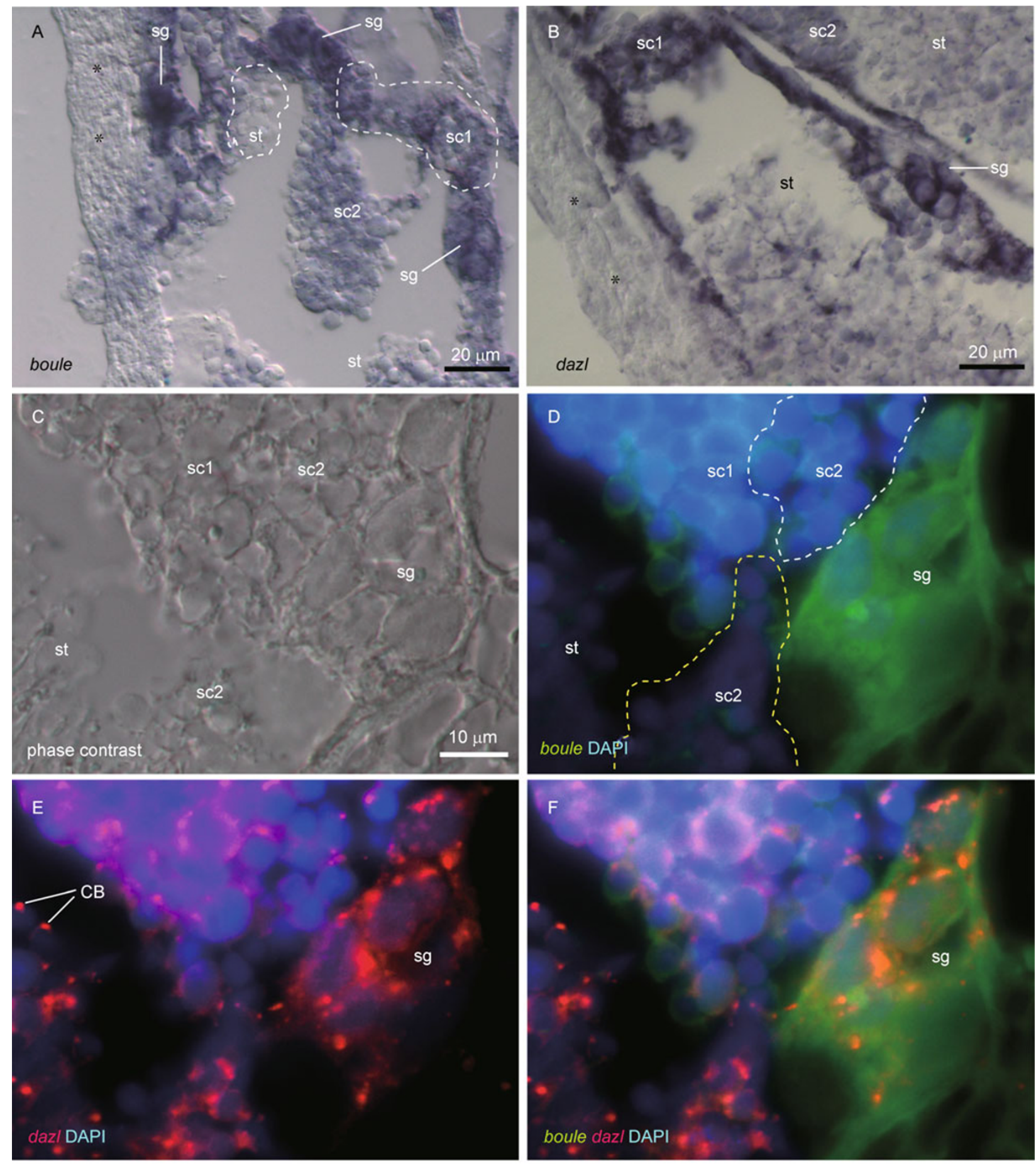

Figure 1. Expression of boule and dazl transcripts in the adult trout testis. (A and B) Chromogenic ISH with antisense probe boule (A) and dazl (B). (C-F) Fluorescence ISH with antisense boule (green) and dazl (red) probes. Spermatogonia are dazI $/$ boule ${ }^{+}$. sg, spermatogonia; sc1, primary spermatocytes; sc2, secondary spermatocytes; st, spermatids; CB, chromatoid body; asterisk, somatic cells, ISH, in situ hybridization. 

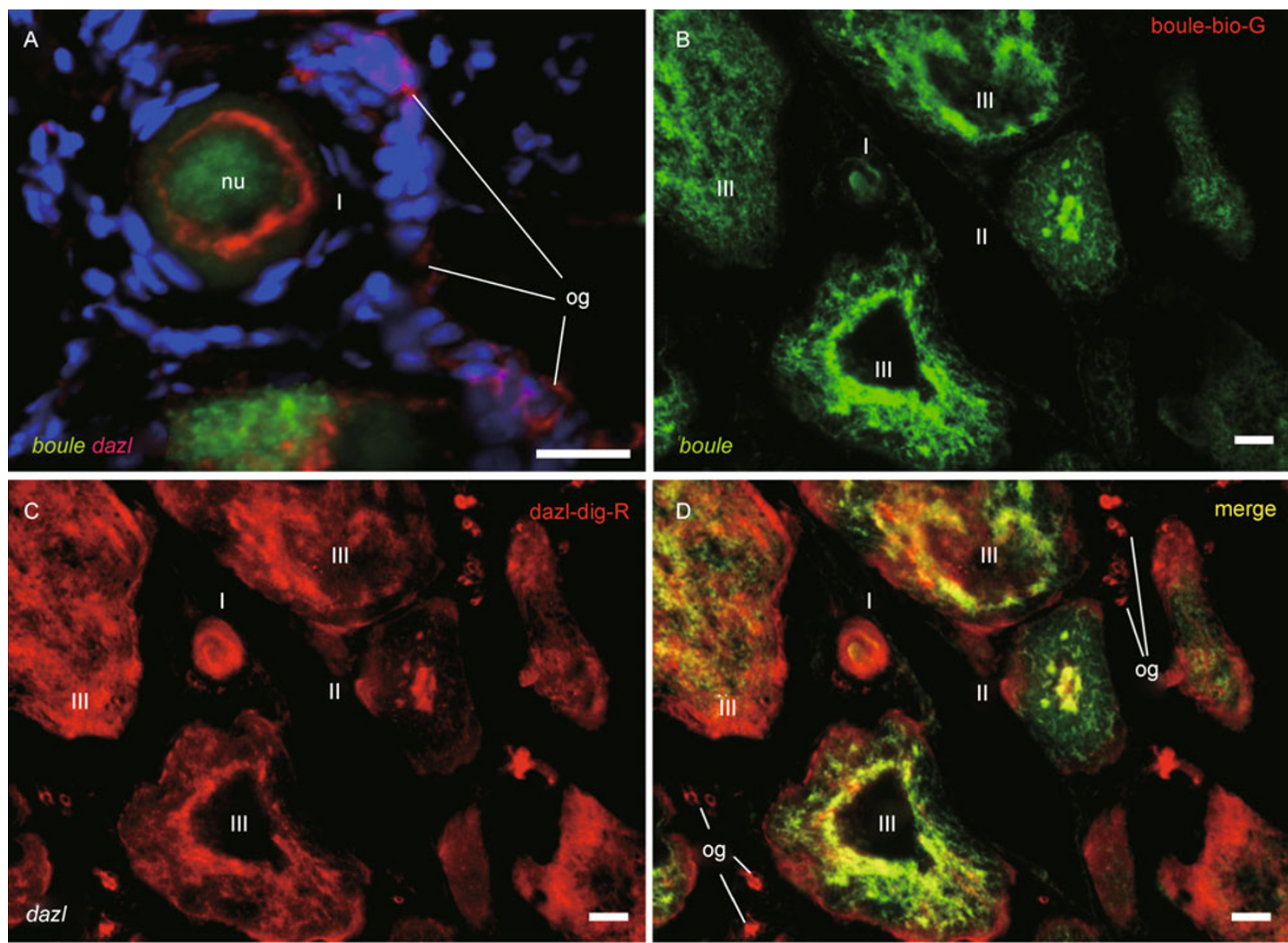

Figure 2. Expression of boule and dazl transcripts in the ovary. (A) One-year-old young ovary after fluorescence ISH with boule (green) and dazl (red) probes. (B-D) Three-year-old adult ovary after fluorescence ISH with boule (green) and vasa (red) probes. Oogonia (og) are dazl'/boule ${ }^{-}$. ISH, in situ hybridization. Scale bars, $50 \mu \mathrm{m}$.

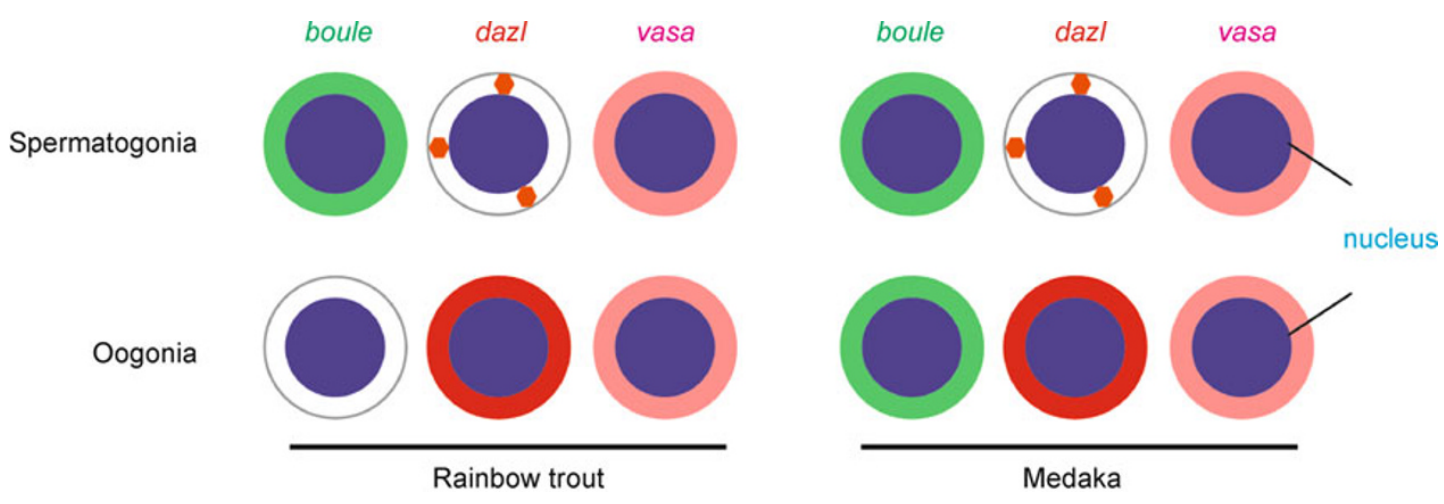

Figure 3. Gonial sex. In rainbow trout and medaka, vasa expression and homogeneous distribution are seen in both male and female gonia, dazl expression is seen also in both male and female gonia but shows different distribution. Strikingly, boule shows expression and uniform distribution in both male and female gonia of medaka, but exhibits sex-specific expression only in male gonia of rainbow trout. In both rainbow trout and medaka, vasa expression in gonia does not show sex dimorphism. Data for rainbow trout are from the present study and (Li et al., 2011 In press), and data for medaka are according to (Xu et al., 2009). 
Together with transplantation data (Okutsu et al., 2006; Yoshizaki et al., 2010), our finding suggests the plasticity of gonial sexuality for reprogramming into both sexes under the influence of the gonadal sex. Our findings suggest that sperm/ egg decision and mitosis/meiosis decision are two independent events, and that sperm/egg decision appears to precede mitosis/meiosis decision.

We have shown that boule lacks expression in oogonia of both immature and mature ovaries. It will be interesting to determine in the future whether boule expression occurs in sexually indeterminate PGCs, or whether its sex-specific expression commences concurrently with gonadal/organismal sex differentiation. Although our study cannot reveal the role of boule and dazl in germ cell sex establishment, the ability to identify the germ cell sexuality prior to meiosis opens new avenues for studying sex differentiation directly in germ cells themselves.

In many organisms, boule expression has been reported to be meiotic in one sex (Xu et al., 2009; Li et al., 2011). One exception is medaka, where boule expression occurs in PGCs, mitotic and meiotic germ cells of both sexes (Xu et al., 2009). Therefore, the male-specific gonial expression of boule is not an evolutionarily conserved premeiotic germ cell marker. This situation appears to parallel the enormous diversity of somatic sex determination. For example, dmyl dmrt1y acts as a male determining gene in medaka (Matsuda et al., 2002; Nanda et al., 2002), which is, however, absent in other species of the genus Oryzias to which medaka belongs (Kondo et al., 2003); and sox9 is a male-sex-determining gene in mammals but does not show sex-dimorphic expression in medaka (Nakamoto et al., 2005). The diversity of regulators governing sperm/egg decision and male/female decision provides the complexity to identify premeiotic germ cell sex in different organisms. Among germ cell markers examined so far, boule exhibits exceptional variation in its expression, ranging from male/female meiotic expression to bisexual mitotic and meiotic expression (Xu et al., 2009; Li et al., 2011). In this regard, it is not surprising that boule becomes a gonial sex marker in one fish but not in another. Whether boule and dazl show sex-specific expression or intracellular distribution in other fish species remains unknown. Our data make rainbow trout a prototype organism to study mechanisms underlying germ cell sexuality in a vertebrate.

\section{CONCLUSION}

We revealed differential expression of boule and differential intracellular distribution of dazl in premeiotic germ cells between rainbow trout male and female, providing convincing evidence for gonial sex in rainbow trout. This finding demonstrates sperm/egg and mitosis/meiosis decisions as two independent events, and puts germ cell sex ahead of mitosis/meiosis switch.

\section{MATERIALS AND METHODS}

\section{Fish and chemicals}

This study was carried out in strict accordance with the recommendations in the Guide for the Care and Use of Laboratory Animals of the National Advisory Committee for Laboratory Animal Research in Singapore and approved by this committee (Permit Number: 27/09). Rainbow trout females were purchased from a fish farmer in Würzburg, Germany, and adult testis samples were kindly provided by Dr. Bo Ma (the Heilongjiang Fisheries Research Institute, Ha'erbing City, China). Chemicals and enzymes were from Sigma and Promega, respectively, unless otherwise indicated.

\section{Sections}

The trout testis and ovary were fixed overnight or longer at $4 \%$ paraformaldehyde (PFA) in phosphate-buffered saline (PBS) at $4^{\circ} \mathrm{C}$. They were dehydrated by passing through an increasing series of ethanol $(70 \%, 80 \%, 90 \%$ and $100 \%)$ for $1 \mathrm{~h}$ each. After an additional treatment in $100 \%$ ethanol, the samples were treated for 30 min each in a mix of Histoclear:ethanol (1:1) and 100\% Histoclear. After a 30 -min incubation in paraffin at $60^{\circ} \mathrm{C}$, the samples were treated overnight in paraffin at $60^{\circ} \mathrm{C}$ and embedded in paraffin. The testis was cut at $5 \mu \mathrm{m}$ and ovary at $8 \mu \mathrm{m}$ on the Leica RM2135 Microtomes (Leica, Germany). Slides of sections were dewaxed by immersion in xylene with three changes, each for $10 \mathrm{~min}$. After rehydration through a descending ethanol series $(100 \%, 90 \%, 70 \%, 50 \%$ and $30 \%)$, the slides were subjected to treatments twice with PBS containing $0.1 \%$ Tween-20 (PBST) for $5 \mathrm{~min}$ each, once with $0.1 \mathrm{M} \mathrm{HCl}$ for $5 \mathrm{~min}$ and three washes in PBST for 5 min each. The samples were treated with proteinase $\mathrm{K}(10 \mu \mathrm{g} / \mathrm{mL})$ for $30 \mathrm{~min}$ at $37^{\circ} \mathrm{C}$, washed three times in PBST for $5 \mathrm{~min}$ each, and refixed in $4 \%$ for $15 \mathrm{~min}$ at room temperature. After three PBST rinses, the samples were subjected to in situ hybridization.

\section{Chromogenic in situ hybridization}

Chromogenic ISH by using BCIP/NBT as substrates was performed as described previously with minor modifications (Xu et al., 2005). Briefly, pOMbol, pOMdazl and pOMvasa were linearized with Xho I and Sac II and used for the synthesis of sense and anti-sense RNA probes from SP6 or T7 promoter by using the digoxigenin (DIG) Labeling Kit (Roche) for dazl and vasa, or the FITC RNA Labeling Kit (Roche) for boule (Li et al., 2011). The RNA probes were treated with RNase-free TURBO DNase (Ambion) and purified by using the $\mathrm{LiCl}$ method according to the supplier's instruction (cat \#AM1340, Ambion). DIG probe and FITC probes were denatured and incubated at $90^{\circ} \mathrm{C}$ for $5 \mathrm{~min}$ followed by rapid cooling on ice. Chromogenic ISH was performed as described previously (Xu et al., 2005). Briefly, the probe was added at $\sim 1 \mu \mathrm{g} / \mathrm{mL}$ to the hybridization buffer $(50 \%$ formamide, $5 \times$ SSC, $500 \mu \mathrm{g} / \mathrm{mL}$ yeast tRNA, $0.1 \%$ Tween-20 and $50 \mu \mathrm{g} / \mathrm{mL}$ heparin). The samples on each slide were covered with $100 \mu \mathrm{L}$ of the hybridization buffer containing a probe and wrapped in parafilm. The slides were put into a Petri dish layered at bottom a Whatmann paper presoaked with $2 \times$ SSC containing $0.1 \%$ Tween-20 (SSCT) and $50 \%$ formamide. The dish was sealed with parafilm and 
incubated overnight at $65^{\circ} \mathrm{C}$. The slides were washed twice in $2 \times$ SSCT- $50 \%$ formamide for 30 min each, twice in $2 \times$ SSCT and twice in $0.2 \times$ SSCT for 30 min each. After blocking for $1 \mathrm{~h}$ in PBST containing $10 \%$ goat serum at room temperature, the samples were incubated with either the alkaline phosphatase (AP) conjugated anti-DIGantibody or AP-conjugated anti-FITC-antibody (Sigma) at 1:2000 in the blocking buffer (PBST containing $2 \%$ blocking reagent and $10 \%$ heat-inactivated fetal bovine serum) for $2 \mathrm{~h}$ at room temperature. Following six PBST washes and preincubation in the pre-staining buffer (NTMT; $100 \mathrm{mM} \mathrm{NaCl}, 50 \mathrm{mM} \mathrm{MgCl}_{2}, 100 \mathrm{mM}$ Tris-Cl, pH 9.5, $0.1 \%$ Tween-20) for $30 \mathrm{~min}$, the samples were incubated in the staining buffer $(0.1 \mathrm{mg} / \mathrm{mL}$ NBT/BCIP in NTMT) in darkness at room temperature for $30-60 \mathrm{~min}$ or at $4^{\circ} \mathrm{C}$ overnight. Color development was microscopically monitored at regular intervals. Nuclear staining was done by using 4',6-Diamidino-2-phenylindole (DAPI; $1 \mu \mathrm{g} / \mathrm{mL}$ ) and embedded in the Gold Antifade reagent (Invitrogen) for microscopy.

\section{Fluorescence in situ hybridization}

Two-color fluorescence in situ hybridization (FISH) (Campbell et al., 2007) was performed by using the tyramide signal amplification $\left(\mathrm{TSA}^{\mathrm{TM}}\right)$ Plus Fluorescence Systems according to the manufacturer's instruction (NEL756, PerkinElmer Life Science)(Xu et al., 2009). Briefly, after hybridization and blocking as described above, the samples were incubated with the peroxidase (POD)-conjugated antiFITC-antibody (Sigma) at a 12000 dilution for $2 \mathrm{~h}$ at room temperature to detect the FITC-labeled probes. Following six PBST washes the samples were incubated for $30 \mathrm{~min}$ in the TSA-Fluorecein at a 1:100 dilution in the TSA Amplification Buffer $\left(0.004 \% \mathrm{H}_{2} \mathrm{O}_{2}\right.$ in $0.1 \mathrm{M}$ borate buffer, $\mathrm{pH}$ 8). The samples were then subjected to detection of the DIG-labeled probe: They were treated for $1 \mathrm{~h}$ in $1 \%$ $\mathrm{H}_{2} \mathrm{O}_{2}$ and incubated for two hours with the horseradish PODconjugated anti-DIG antibody (Sigma) at a 1:2000 dilution, followed by a $30-\mathrm{min}$ incubation in TSA-Cy3 for $30 \mathrm{~min}$. The samples were finally stained for nuclei by using DAPI and embedded in the Gold Antifade reagent (Invitrogen) for microscopy. In this FISH procedure, two differently labeled antisense RNA probes are co-incubated with a sample in the hybridization step and subjected to sequential color development into green and red fluorescence, and nuclei are stained with DAPI for blue fluorescence. This procedure features multiple combinations between bright-field optics and three colors of fluorescent optics, allowing for precise comparisons of the relative signal intensity and distribution of different molecules. FISH in medaka provides detection sensitivity $\sim 100$ times that of the chromogenic ISH (Liu et al., 2009; Xu et al., 2009).

\section{Microscopy and photography}

Observation and photography under a Leica MZFIII stereo microscope, Zeiss Axiovertinvert and Axiovert upright microscopes with a Zeiss AxioCam M5Rc digital camera (Zeiss Corp) were as described previously (Xu et al., 2007).

\section{ACKNOWLEDGEMENTS}

We thank J. Deng for collecting fish samples. This work was supported by the Biomedical Research Council of Singapore (R-081-21-19-585 \& SBIC-SSCC C-002-2007).

\section{ABBREVIATIONS}

AP, alkaline phosphatase; CB, chromatoid body; DAPI, 4',6-Diamidino-2-phenylindole; FISH, fluorescence in situ hybridization; ISH, in situ hybridization; PBS, phosphate-buffered saline; PFA, paraformaldehyde; POD, peroxidase

\section{REFERENCES}

Baron, D., Houlgatte, R., Fostier, A., and Guiguen, Y. (2005). Largescale temporal gene expression profiling during gonadal differentiation and early gametogenesis in rainbow trout. Biol Reprod 73, 959-966.

Campbell, K.H., Fisher, P., Chen, W.C., Choi, I., Kelly, R.D., Lee, J.H., and Xhu, J. (2007). Somatic cell nuclear transfer: Past, present and future perspectives. Theriogenology 68, S214-S231.

Herpin, A., Rohr, S., Riedel, D., Kluever, N., Raz, E., and Schartl, M. (2007). Specification of primordial germ cells in medaka (Oryzias latipes). BMC Dev Biol 7, 3.

Hong, Y., Liu, T., Zhao, H., Xu, H., Wang, W., Liu, R., Chen, T., Deng, J., and Gui, J. (2004). Establishment of a normal medakafish spermatogonial cell line capable of sperm production in vitro. Proc Natl Acad Sci U S A 101, 8011-8016.

Hong, Y., Winkler, C., and Schartl, M. (1996). Pluripotency and differentiation of embryonic stem cell lines from the medakafish (Oryzias latipes). Mech Dev 60, 33-44.

Hong, Y., Winkler, C., and Schartl, M. (1998). Production of medakafish chimeras from a stable embryonic stem cell line. Proc Natl Acad Sci U S A 95, 3679-3684.

Kimble, J., and Page, D.C. (2007). The mysteries of sexual identity. The germ cell's perspective. Science 316, 400-401.

Kocer, A., Reichmann, J., Best, D., and Adams, I.R. (2009). Germ cell sex determination in mammals. Mol Hum Reprod 15, 205-213.

Kondo, M., Nanda, I., Hornung, U., Asakawa, S., Shimizu, N., Mitani, H., Schmid, M., Shima, A., and Schartl, M. (2003). Absence of the candidate male sex-determining gene dmrt $1 \mathrm{~b}(\mathrm{Y})$ of medaka from other fish species. Curr Biol 13, 416-420.

Kurokawa, H., Saito, D., Nakamura, S., Katoh-Fukui, Y., Ohta, K., Baba, T., Morohashi, K., and Tanaka, M. (2007). Germ cells are essential for sexual dimorphism in the medaka gonad. Proc Natl Acad Sci U S A 104, 16958-16963.

Li, M., Hong, N., Xu, H., Yi, M., Li, C., Gui, J., and Hong, Y. (2009). Medaka vasa is required for migration but not survival of primordial germ cells. Mech Dev 126, 366-381.

Li, M., Shen, Q., Xu, H., Wong, F.M., Cui, J., Li, Z., Hong, N., Wang, L., Zhao, H., Ma, B., et al. (2011). Differential conservation and divergence of fertility genes boule and dazl in the rainbow trout. PloS ONE. (In press)

Liu, L., Hong, N., Xu, H., Li, M., Yan, Y., Purwanti, Y., Yi, M., Li, Z., Wang, L., and Hong, Y. (2009). Medaka dead end encodes a cytoplasmic protein and identifies embryonic and adult germ cells. Gene Expr Patterns 9, 541-548.

Matsuda, M., Nagahama, Y., Shinomiya, A., Sato, T., Matsuda, C., Kobayashi, T., Morrey, C.E., Shibata, N., Asakawa, S., Shimizu, N., et al. (2002). DMY is a Y-specific DM-domain gene required for male development in the medaka fish. Nature 417, 559-563.

Nakamoto, M., Suzuki, A., Matsuda, M., Nagahama, Y., and Shibata, N. (2005). Testicular type Sox 9 is not involved in sex determination but might be in the development of testicular structures in the 
medaka, Oryzias latipes. Biochem Biophys Res Commun 333, 729-736.

Nanda, I., Kondo, M., Hornung, U., Asakawa, S., Winkler, C., Shimizu, A., Shan, Z., Haaf, T., Shimizu, N., Shima, A., et al. (2002). A duplicated copy of DMRT1 in the sex-determining region of the $\mathrm{Y}$ chromosome of the medaka, Oryzias latipes. Proc Natl Acad Sci U S A 99, 11778-11783.

Okutsu, T., Shikina, S., Kanno, M., Takeuchi, Y., and Yoshizaki, G. (2007). Production of trout offspring from triploid salmon parents. Science 317, 1517.

Okutsu, T., Suzuki, K., Takeuchi, Y., Takeuchi, T., and Yoshizaki, G. (2006). Testicular germ cells can colonize sexually undifferentiated embryonic gonad and produce functional eggs in fish. Proc Natl Acad Sci U S A 103, 2725-2729.

Uhlenhaut, N.H., Jakob, S., Anlag, K., Eisenberger, T., Sekido, R., Kress, J., Treier, A.C., Klugmann, C., Klasen, C., Holter, N.I., et al. (2009). Somatic sex reprogramming of adult ovaries to testes by FOXL2 ablation. Cell 139, 1130-1142.

Vizziano, D., Randuineau, G., Baron, D., Cauty, C., and Guiguen, Y. (2007). Characterization of early molecular sex differentiation in rainbow trout, Oncorhynchus mykiss. Dev Dyn 236, 2198-2206.
Volff, J.N., Nanda, I., Schmid, M., and Schartl, M. (2007). Governing sex determination in fish: regulatory putsches and ephemeral dictators. Sex Dev 1, 85-99.

Wylie, C. (1999). Germ cells. Cell 96, 165-174.

$\mathrm{Xu}, \mathrm{H}$., Gui, J., and Hong, Y. (2005). Differential expression of vasa RNA and protein during spermatogenesis and oogenesis in the gibel carp (Carassius auratus gibelio), a bisexually and gynogenetically reproducing vertebrate. Dev Dyn 233, 872-882.

Xu, H., Li, M., Gui, J., and Hong, Y. (2007). Cloning and expression of medaka dazl during embryogenesis and gametogenesis. Gene Expr Patterns 7, 332-338.

Xu, H., Li, M., Gui, J., and Hong, Y. (2010). Fish germ cells. Sci China Life Sci 53, $435-446$.

Xu, H., Li, Z., Li, M., Wang, L., and Hong, Y. (2009). Boule is present in fish and bisexually expressed in adult and embryonic germ cells of medaka. PLoS One 4, e6097.

Yi, M., Hong, N., and Hong, Y. (2009). Generation of medaka fish haploid embryonic stem cells. Science 326, 430-433.

Yoshizaki, G., Ichikawa, M., Hayashi, M., Iwasaki, Y., Miwa, M., Shikina, S., and Okutsu, T. (2010). Sexual plasticity of ovarian germ cells in rainbow trout. Development 137, 1227-1230. 